\title{
The potential energy of non-resonant optimal bianisotropic particles in an electromagnetic field does not depend on time
}

\author{
Igor Semchenko $^{1}$, Sergei Khakhomov ${ }^{1, *}$, Alexey Balmakou ${ }^{1}$, and Sergei Tretyakov ${ }^{2}$ \\ 1 Department of Physics, Gomel State University, Sovetskaya Str. 104, 246019 Gomel, Belarus \\ 2 Department of Radio Science and Engineering, Aalto University, Aalto FI 00076, Finland
}

Received 25 February 2014 / Accepted 19 May 2014

\begin{abstract}
In this work, we consider potential energy of recently conceptualized optimal (balanced) bianisotropic particles in electromagnetic fields. The case of non-resonant lossless particles is studied. Knowing the potential energy of optimal bianisotropic particles in the fields of the respective excitations, we find the acting force on the particle in inhomogeneous external fields. It is found that for optimal particles with the balanced values of the polarizabilities the potential energy and acting force are time independent.
\end{abstract}

Key words: Bianisotropic particle, Electromagnetic wave, Potential energy.

\section{Introduction}

Let us consider an electrically small bianisotropic particle which has interconnected electric dipole and magnetic moments. These moments can be written in terms of the exciting fields as:

$$
\begin{gathered}
\vec{p}=\varepsilon_{0} \alpha_{e e} \vec{E}-j \sqrt{\varepsilon_{0} \mu_{0}} \alpha_{e m} \stackrel{\leftarrow}{H}, \\
\vec{m}=\alpha_{m m} \vec{H}+j \sqrt{\frac{\varepsilon_{0}}{\mu_{0}}} \alpha_{m e} \vec{E} .
\end{gathered}
$$

From the principle of symmetry of the kinetic coefficients it follows that the polarizabilities of reciprocal particles satisfy:

$$
\begin{aligned}
& \alpha_{e e}^{(i j)}=\alpha_{e e}^{(j i)}, \\
& \alpha_{m m}^{(i j)}=\alpha_{m m}^{(j i)}, \\
& \alpha_{m e}^{(i j)}=\alpha_{e m}^{(j i)} .
\end{aligned}
$$

Reciprocal bianisotropic particles can be conveniently classified based on the symmetry properties of the magnetoelectric coefficients. The case of symmetric matrices $\alpha_{m e}^{(i j)}=$ $\alpha_{m e}^{(j i)}, \alpha_{e m}^{(i j)}=\alpha_{e m}^{(i i)}$ correspond to chiral particles, while the case of antisymmetric matrices $\alpha_{m e}^{(i j)}=-\alpha_{m e}^{(i i)}, \alpha_{e m}^{(i j)}=-\alpha_{e m}^{(j i)}$ corresponds to omega media [1]. Recently, it was found that balanced particles, such that all the polarizablities have equal strength, possess very special, and extreme properties.
They store maximum (or minimum, equal to zero) reactive energy in given fields [2], they are "invisible" for certain excitations [3], they present the optimal configurations for extracting energy from external fields [4]. Planar arrays of balanced particles realize optimal configurations for polarization transformers [5-7] or reflectors with controllable reflection phase [8]. Furthermore, arrays of balanced nonreciprocal bianisotropic particles can realize single-particle-thick ideal isolators $[9,10]$.

In this paper we consider another aspect of the extreme properties of balanced bianisotropic particles: we study the potential energy of the particles in given fields and consider the force acting on the particles in nonuniform fields. The study is limited to the case of non-resonant lossless particles. We find that if the polarizabilties are balanced and the particle interacts with the incident fields optimally strong, the potential energy is time-independent. This implies that the mechanical force generated by time-harmonic fields is time-independent. Of course, when external field of the orthogonal polarization acts, the particle is not excited at all, implying that the force acting on the particle is identically zero. We think that this interesting property can have important implications in nanotechnologies and nanomedicine.

\section{Basic assumptions and relations}

Assume that the influence of the fields on the particle which is not of a resonant character and losses are negligible, therefore all the polarizabilities $\alpha_{e e}, \alpha_{m m}, \alpha_{e m}$ are real quantities:

$$
\alpha_{e e}=\alpha_{e e}^{*}, \alpha_{m m}=\alpha_{m m}^{*}, \alpha_{e m}=\alpha_{e m}^{*} .
$$


In this case we can neglect the dissipation of electromagnetic wave energy and consider the interaction of the particle with the electromagnetic field as quasi-stationary. We can introduce the potential energy of the bianisotropic particle in an electromagnetic field:

$$
W=-\vec{p}^{\prime} \vec{E}^{\prime}-\vec{m}^{\prime} \vec{B}^{\prime},
$$

where primes denote the real parts of the vectors $\vec{p}, \vec{m}, \vec{E}$, and $\vec{B}$, multiplied by the time-dependence factor $\exp (j \omega t)$. Let us start the analysis of the case of a chiral particle, which interacts optimally with circularly polarized waves [2-4]. Consider the interaction of bianisotropic particle with a spherical left-handed circularly polarized wave. Dependence of the wave vector $\vec{E}$ on the coordinate $z$ can be written as:

$$
\vec{E}_{-}=\frac{1}{z} E_{0} \frac{\vec{x}_{0}-j \vec{y}_{0}}{\sqrt{2}} \exp [j(\omega t-k z)] .
$$

The electric field vector of such a wave forms left-handed screw in space, which gives the reason to call it "left-handed" circularly polarized wave. At the same time for an observer looking along the wave vector, the vector $\vec{E}$ rotates with time in a clockwise direction. Therefore, according to another definition, such a wave is called "right" circularly polarized. For such a wave in free space the magnetic field vectors have the form:

$$
\vec{B}_{-}=\frac{j}{c} \vec{E}_{-}, \quad \vec{H}_{-}=\frac{1}{\mu_{0}} \vec{B}_{-} .
$$

\section{Potential energy}

Without loss of generality, we assume that the electric and magnetic moments generated in a chiral particle under the influence of electromagnetic waves have only the $x$-components. In this case, we can calculate the potential energy in equation (7) as:

$$
W=p_{x}^{\prime} E_{x}^{\prime}-m_{x}^{\prime} B_{x}^{\prime}
$$

It can be written as:

$$
W=\langle W\rangle+\Delta W,
$$

where $\langle W\rangle$ is the time-average value of the potential energy, and $\Delta W$ is the time-dependent part of the potential energy. After simple calculations, we obtain:

$$
\begin{gathered}
\langle W\rangle=-\frac{1}{4} \varepsilon_{0} \frac{E_{0}^{2}}{z^{2}}\left(\alpha_{e e}^{(11)}+\alpha_{m m}^{(11)}+2 \alpha_{e m}^{(11)}\right), \\
\Delta W=-\frac{1}{4} \varepsilon_{0} \frac{E_{0}^{2}}{z^{2}}\left(\alpha_{e e}^{(11)}-\alpha_{m m}^{(11)}\right) \cos (2 \omega t-2 k z),
\end{gathered}
$$

here superindex 1 denotes axis $x$. The behavior of the socalled optimal or balanced particle is of particular interest, because for the particle the polarizabilities satisfy the following expressions:

$$
\begin{gathered}
\alpha_{e e}=\alpha_{m m}, \\
\alpha_{e e}= \pm \alpha_{e m} .
\end{gathered}
$$

If equation (15) holds with the sign "+", we obtain:

$$
\langle W\rangle=-\varepsilon_{0} \frac{E_{0}^{2}}{z^{2}} \alpha_{e e}^{(11)},
$$

$$
\Delta W=0 .
$$

This means that the potential energy of a particle in this electromagnetic field is independent of time, although the particle is affected by the time-harmonic field. If equation (15) holds with the sign "-", we find:

$$
\langle W\rangle=0, \quad \Delta W=0 .
$$

Hence, such a particle does not interact with the considered circularly polarized wave, as it is known for balanced chiral particles $[2,3]$. We see that the properties of the particle have a selective action relatively to the wave polarization, i.e., to the direction of rotation of the vector $\vec{E}$ of the wave.

\section{Acting force}

Since the potential energy of the optimal particle in the field of a circularly polarized wave does not depend on time, we can calculate a force, acting on the particle in the inhomogeneous field of a spherical wave. This force in the quasi-static approximation is equal:

$$
F=-\nabla W
$$

Using equations (16) and (19), we obtain the projection of force on the $z$-axis:

$$
F_{z}=-2 \varepsilon_{0} \frac{E_{0}^{2}}{z^{3}} \alpha_{e e}^{(11)} .
$$

Consequently, the optimal particle is attracted to the source radiating a spherical circularly polarized wave and the force of attraction does not depend on time.

If we apply the formula (19) in the general case for chiral particles not having optimal parameters, the expression for the $z$-component of the force can be written as:

$$
\begin{gathered}
F_{z}=-\frac{1}{2} \varepsilon_{0} \frac{E_{0}^{2}}{z^{3}}\left(\alpha_{e e}^{(11)}+\alpha_{m m}^{(11)}+2 \alpha_{e m}^{(11)}\right) \\
-\frac{1}{2} \varepsilon_{0} \frac{E_{0}^{2}}{z^{2}}\left(\alpha_{e e}^{(11)}-\alpha_{m m}^{(11)}\right)\left(\frac{1}{z} \cos (2 \omega t-2 k z)-k \sin (2 \omega t-2 k z)\right) .
\end{gathered}
$$

Figures 1 and 2 plot the dependence of the potential energy and force on time for various non-resonant bianisotropic chiral particles: dielectric, paramagnetic, and having optimal parameters.

The graphs show that the optimal chiral particle with a positive magnetoelectric parameter $\alpha_{e m}$ experiences stable attraction to the source of a left-handed circularly polarized wave. The force of attraction does not depend on time. If the bianisotropic particle is optimal, but has a negative magnetoelectric parameter $\alpha_{e m}$, the interaction of particle with the left-handed circularly polarized wave is absent. However, for particles with 


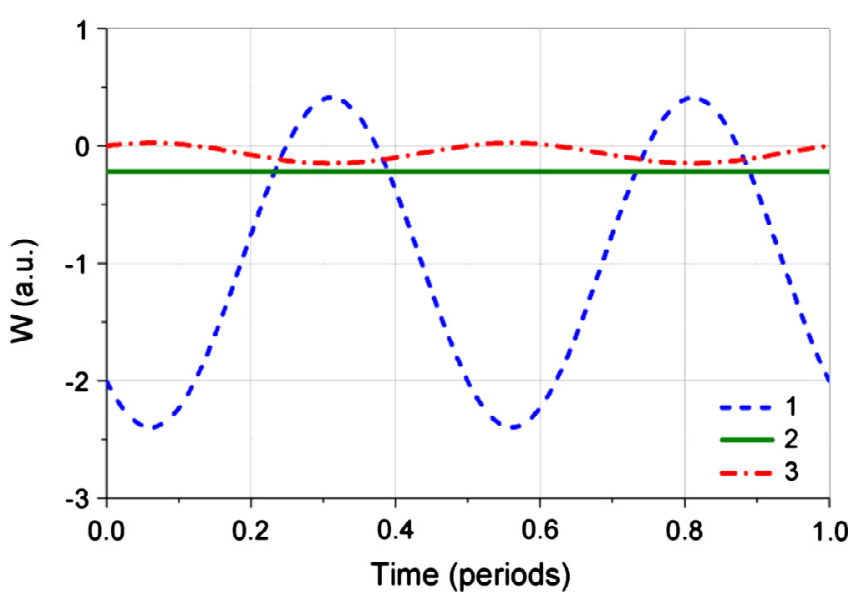

Figure 1. Potential energy of chiral particles vs. time: the blue dashed line (no. 1) corresponds to a particle with $\alpha_{e e} \neq 0, \alpha_{m m}=0$, $\alpha_{e m}=0$. The red dash-dot line (no. 3) corresponds to the particle with $\alpha_{m m} \neq 0, \alpha_{e e}=0, \alpha_{e m}=0$. The green solid line (no. 2) represents the particle with $\alpha_{e e}=\alpha_{m m}=\alpha_{e m} \neq 0$.

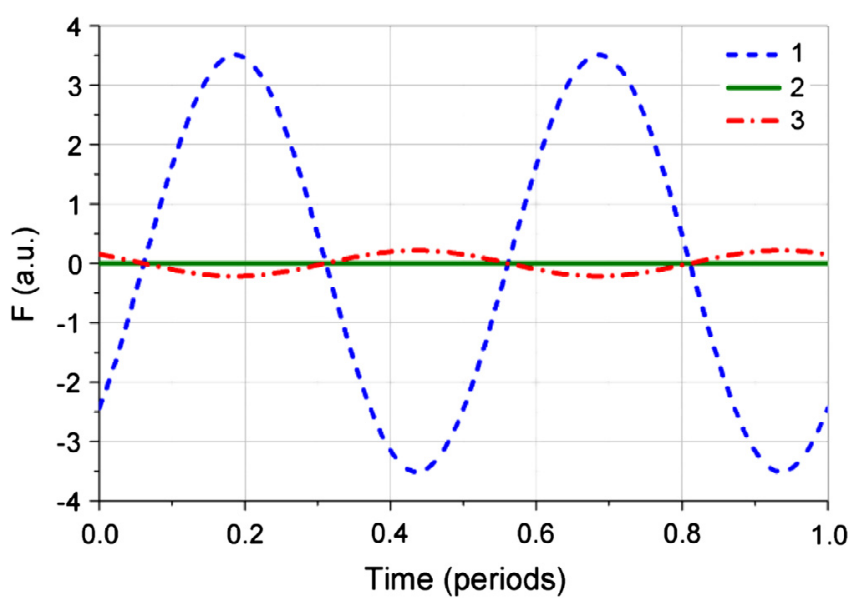

Figure 2. Force acting on chiral particles vs. time: the blue dashed line (no. 1) corresponds to the particle with $\alpha_{e e} \neq 0, \alpha_{m m}=0$, $\alpha_{e m}=0$. The red dash-dot line (no. 3) corresponds to the particle with $\alpha_{m m} \neq 0, \alpha_{e e}=0, \alpha_{e m}=0$. The green solid line (no. 2) represents the particle with $\alpha_{e e}=\alpha_{m m}=\alpha_{e m} \neq 0$.

non-optimal parameters, the attraction force is time-dependent with varying sign. For example, if the particle is purely dielectric or purely paramagnetic, the amplitude variations of the force are much greater than the average force.

It is easy to show that the additional term in the expression for the force which appears in the Abraham's approach to the definition of momentum of electromagnetic field $\vec{F}=\mathrm{d} / \mathrm{d} t\left(\vec{p} \times \vec{B}+(\vec{m} \times \vec{E}) / c^{2}\right)$ [11] equals zero when averaged over time.

\section{Generalizations}

In fact the same conclusion holds for balanced particles of the other fundamental reciprocal class: omega particles [1]. In this case, relations (3)-(5) hold, but the magnetoelectric dyadics (tensors) are anti-symmetric. Any anti-symmetric tensor can be presented as a vector product of a vector with a unit dyadic:

$$
\alpha_{m e}=-\alpha_{e m}=\Omega \vec{z}_{0} \times I
$$

Here $I$ is the unit dyadic and for the case of non-resonant lossless particles the coupling coefficient $\Omega$ is real. Omega particles interact optimally with evanescent linearly polarized fields. Thus, we assume that the incident field is linearly polarized (electric field is along the $x$ axis, and magnetic field is along the $y$ axis), and the wave impedance is purely imaginary:

$$
\begin{gathered}
\vec{E}=\frac{1}{z} E_{0} \exp [j(\omega t-k z)] \vec{x}_{0}, \quad \vec{B}=\frac{1}{j c} \vec{z}_{0} \times \vec{E}, \\
\vec{H}=\frac{1}{\mu_{0}} \vec{B} .
\end{gathered}
$$

Without loss of generality, we can assume that the electric dipole moment induced in the particle is along the axis $x$, and the magnetic dipole moment is along $y$. Substituting into the expression for the potential energy in equation (10), we find, similarly to the case of chiral particles,

$$
\begin{gathered}
\langle W\rangle=-\frac{1}{4} \varepsilon_{0} \frac{E_{0}^{2}}{z^{2}}\left(\alpha_{e e}+\alpha_{m m}+2 \Omega\right), \\
\Delta W=-\frac{1}{4} \varepsilon_{0} \frac{E_{0}^{2}}{z^{2}}\left(\alpha_{e e}-\alpha_{m m}\right) \cos (2 \omega t-2 k z) .
\end{gathered}
$$

Obviously, the same conclusions regarding timeindependence of the potential energy and acting force in nonuniform fields hold also for balanced omega particles. Likewise we see that changing sign of the coupling coefficient (or reversing the direction of evanescent wave decay) of a balanced particle makes $\langle W\rangle=0$, which corresponds to the invisibility regime [3]. Another interesting conclusion is that the timevarying part of the potential energy does not depend on the coupling coefficient only if the particle has magneto-electric coupling of only one type: chiral or omega, and if the excitation polarization is of the respective type. If both chirality parameter and the omega coefficient are not zero and the external field is arbitrary, this potential energy depends on time even if all the parameters are balanced.

Further generalization can be made for nonreciprocal bianisotropic particles. The two fundamental nonreciprocal classes are moving media, with:

$$
\alpha_{m e}=\alpha_{e m}=j V \vec{z}_{0} \times I,
$$

and Tellegen media, with:

$$
\alpha_{m e}=-\alpha_{e m}=j \chi .
$$

Moving media interact optimally with linearly polarized propagating plane waves. Thus, we can assume that the incident fields are:

$$
\begin{gathered}
\vec{E}=\frac{1}{z} E_{0} \exp [j(\omega t-k z)] \vec{x}_{0}, \quad \vec{B}=\frac{1}{c} \vec{z}_{0} \times \vec{E}, \\
\vec{H}=\frac{1}{\mu_{0}} \vec{B} .
\end{gathered}
$$


Writing again the expression for the potential energy, we come to a different conclusion: because all the dipole moments and fields coming into expression (10) are now in phase, the time-averaged and time-varying parts of the potential energy are proportional to the same coefficient, equal to $\alpha_{e e}+\alpha_{m m}-2 \mathrm{~V}$. For the balances particle this is zero for one of the possible signs of the "velocity" parameter $V$, which again corresponds to the invisibility regime [3]. However, for the opposite choice of the sign, the potential energy remains time-dependent. It is easy to check that this is true also for the other nonreciprocal class, the Tellegen particle, which is optimal in the fields of circularly polarized standing waves.

\section{Conclusions}

We have considered non-resonant bianisotropic particles in the field of variously polarized electromagnetic waves. In the quasi-stationary approximation the potential energy of a particle in an electromagnetic field is calculated. Bianisotropic particles with the optimal parameters, which are characterized by equally strong electric, magnetic, and magnetoelectric effects, have been in the focus of the study. We have discovered that the potential energy of such particles in appropriately polarized electromagnetic fields is not time dependent. For instance, if a balanced chiral particle is in the field of a circularly polarized spherical wave, the particle is attracted to the source of radiation of the wave with the attraction force which does not depend on time. If the wave changes its polarization to the opposite, then the potential energy of a particle with the optimal parameters and the force acting on it are zero, i.e., particle-field interaction disappears. It is intriguing that this conclusion holds true for both fundamental classes of reciprocal bianisotropic particles, but not for the nonreciprocal ones. We expect that the timeindependence of force applied to a particle in time-harmonic fields can have important applications e.g., in nanoparticle manipulation or separation. This study has been limited to lossless particles with negligible dispersion. For strongly dispersive and lossy particles, such as e.g., resonant helices or omega particles, care should be taken in properly defining the notion of potential energy, similarly to calculations of stored field energy in dispersive and lossy materials.

\section{References}

1. A.N. Serdyukov, I.V. Semchenko, S.A. Tretyakov, A. Sihvola, Electromagnetics of bi-anisotropic materials: Theory and applications, Gordon and Breach Science Publishers, Amsterdam, 2001.

2. I.V. Semchenko, S.A. Khakhomov, A.L. Samofalov, Optimal shape of spiral: Equality of dielectric, magnetic and chiral properties, Proceedings of META'08, NATO Advanced Research Workshop, Metamaterials for Secure Information and Communication Technologies, Marrakesh, Morocco, 2008, pp. 71-80.

3. J. Vehmas, Y. Ra'di, A.O. Karilainen, S. Tretyakov, Eliminating electromagnetic scattering from small particles, IEEE Trans. Antennas Propag. 61 (2013) 3747-3756.

4. Y. Ra'di, S.A. Tretyakov, Balanced and optimal bianisotropic particles: maximizing power extracted from electromagnetic fields, New J. Physics 15 (2013) 053008.

5. I.V. Semchenko, S.A. Khakhomov, A.P. Balmakov, Polarization selectivity of interaction of DNA molecules with X-ray radiation, Biophysics 552 (2010) 194-198.

6. I.V. Semchenko, S.A. Khakhomov, A.L. Samofalov, Transformation of the polarization of electromagnetic waves by helical radiators, J. Commun. Technol. Electron. 528 (2007) 850-855.

7. T. Niemi, A. Karilainen, S. Tretyakov, Synthesis of polarization transformers, IEEE Trans. Antennas Propag. 616 (2013) 3102-3111.

8. Y. Ra'di, V.S. Asadchy, S.A. Tretyakov, Tailoring reflections from thin composite metamirrors, IEEE Trans. Antennas and Propagation 2014.

9. Y. Ra'di, V.S. Asadchy, S.A. Tretyakov, One-way transparent sheets, Phys. Rev. B 89 (2014) 075109-075109.

10. Y. Ra'di, V.S. Asadchy, S.A. Tretyakov, Total absorption of electromagnetic waves in ultimately thin layers, IEEE Trans. Antennas Propag. 619 (2013) 4606-4614.

11. E.A. Hinds, S.M. Barnett, Momentum exchange between light and a single atom: Abraham or Minkowski? Phys. Rev. Lett. 102 (2009) 050403.

Cite this article as: Semchenko I, Khakhomov S, Balmakou A \& Tretyakov S: The potential energy of non-resonant optimal bianisotropic particles in an electromagnetic field does not depend on time. EPJ Appl. Metamat. 2014, 1, 4. 